\title{
Barriers to the Operation of Patient Safety Incident Reporting Systems in Korean General Hospitals
}

\author{
Jee-In Hwang, $\mathrm{PhD}^{1}$, Sang-IL Lee, $\mathrm{MD}, \mathrm{PhD}^{2}$, Hyeoun-Ae Park, $\mathrm{PhD}^{3}$ \\ 'Department of Nursing Management, College of Nursing Science, Kyung Hee University, Seoul; ${ }^{2}$ Department of Preventive Medicine, University of Ulsan \\ College of Medicine, Seoul; ${ }^{3}$ College of Nursing and Research Institute of Nursing Science, Seoul National University, Seoul, Korea
}

Objectives: This study aimed to explore the barriers to and factors facilitating the operation of patient safety incident reporting systems. Methods: A qualitative study that used a methodological triangulation method was conducted. Participants were those who were involved in or responsible for managing incident reporting at hospitals, and they were recruited via a snowballing sampling method. Data were collected via interviews or emails from 42 nurses at 42 general hospitals. A qualitative content analysis was performed to derive the major themes related to barriers to and factors facilitating incident reporting. Results: Participants suggested 96 barriers to incident reporting in their hospitals at the organizational and individual levels. Low reporting rates, especially for near misses, were the most commonly reported issue, followed by poorly designed incident reporting systems and a lack of adequate patient safety leadership by mid-level managers. To resolve and overcome these barriers, 104 recommendations were suggested. The high-priority recommendations included introducing reward systems; improving incident reporting systems, by for instance implementing a variety of reporting channels and ensuring reporter anonymity; and creating a strong safety culture. Conclusions: The barriers to and factors facilitating incident reporting include various organizational and individual factors. As an important way to address these challenging issues and to improve the incident reporting systems in hospitals, we suggest several feasible methods of doing so.

Keywords: Patient Safety, Risk Management

Submitted: November 22, 2012

Revised: 1st, December 17, 2012; 2nd, December 26, 2012

Accepted: December 29, 2012

\section{Corresponding Author}

Hyeoun-Ae Park, PhD

College of Nursing and Research Institute of Nursing Science, Seoul National University, 103 Daehak-ro, Jongno-gu, Seoul 110-799, Korea. Tel: +82-2-740-8827, Fax:+82-2-765-4103, E-mail: hapark@ snu.ac.kr

This is an Open Access article distributed under the terms of the Creative Commons Attribution Non-Commercial License (http://creativecommons.org/licenses/by$\mathrm{nc} / 3.0 /$ ) which permits unrestricted non-commercial use, distribution, and reproduction in any medium, provided the original work is properly cited.

(C) 2012 The Korean Society of Medical Informatics

\section{Introduction}

Continued attention to patient safety has been paid since the U.S. Institute of Medicine reported that a substantial number of patients have died each year due to medical errors [1]. Thereafter, health care organizations have made consistent efforts to ensure patient safety. Incident reporting in health care is considered a way to monitor, prevent, and reduce the occurrence of patient safety events. Although mandatory patient safety incident reporting systems have been introduced at the regional or national levels [2-4], most incident reporting systems in hospitals depend on voluntary reports by staff. Thus, one of the main issues in the operation of in- 
cident reporting systems is how to facilitate staff members' reporting of incidents, including near misses [5].

Several studies have explored the barriers to incident reporting in healthcare settings [3-11]. These barriers are associated with various characteristics at the organizational and individual levels [8]. Evans et al. [5] reported lack of feedback as the most frequently stated barrier to incident reporting. Kreckler et al. [10] suggested that the primary factors that negatively influence incident reporting include lack of understanding about what constitutes a patient safety incident, lack of feedback, lack of time, and fear of blame. In addition, differences in reporting rates according to level of harm $[5,6,10]$ and profession [10] have been found. For instance, less serious incidents and near misses are likely to go underreported $[5,6,10]$, and physicians are less likely to report incidents than nurses $[6,10]$. Braithwaite et al. [4] suggested that the most frequently encountered barriers to reporting incidents are culturally embedded. Kousgaard et al. [3] found that the major reasons for low reporting rates were related to a perceived lack of practical usefulness, issues of time and effort in busy situations, and consideration of other professionals involved.

Korean hospitals generally include the operation of incident reporting systems in their risk management activities. Previous studies conducted in Korea have found that the majority of nurses and physicians are uncomfortable with reporting errors and may often perceive their workplace to have a weak safety culture $[12,13]$. In addition, a study conducted in 2008 reported that hospitals use various forms to collect patient safety incident data, and the most frequent reason for low reporting rates was fear of blame or punishment [14]. The National Healthcare Accreditation Program, launched in 2010, has considerably influenced the implementation of incident reporting systems in Korean hospitals. As this program includes standards for patient safety activities using a hospital-wide patient safety reporting system, hospitals have since introduced or expanded their reporting systems to cover the entire hospital. Furthermore, the computerization of incident reporting systems has been recognized as inevitable for more efficient analysis and management of incidents. Although there are no formal data available on the current status of incident reporting systems, the majority of tertiary hospitals that have computerized hospital information systems, including electronic medical records, have integrated their incident reporting systems with existing hospital information systems.

For the successful implementation of incident reporting systems as patient safety systems, it is important to identify problems in current incident reporting systems, and how they can be improved. Therefore, the purpose of this study was to explore these issues, specifically focusing on the barriers to and facilitating factors in the operation of patient safety incident reporting systems. Since the majority of previous research was quantitative studies and little is known about computerized incident reporting systems in Korea, we used a qualitative approach using methodological triangulation to obtain a better understanding. The results will help hospitals develop strategies for improving incident reporting systems, and can provide insight into what must be considered in the designing of an incident reporting system at the national level.

\section{Methods}

\section{Study Design}

A qualitative research was carried out using methodological triangulation to understand problems in the operation of hospital incident reporting systems, and how they can be improved. The research questions were: 1) What are the problems in or barriers to the operation of incident reporting system in your hospital? 2) What are some measures to resolve or overcome these problems or barriers? This study was a part of a large research project examining patient safety incident reporting systems in general hospitals. The overall study protocol was approved by the Kyung Hee University Ethics Committee (No. 2010-007).

\section{Study Participants and Setting}

The subjects were staff members with special expertise in patient safety incident reporting systems in hospitals. Since they were hard to locate, a snowballing method was used for recruitment; initial informants recommended potential candidates as key informants. Participants were staff members who were involved in or responsible for managing hospitalwide patient safety incident reporting in general hospitals. The final sample consisted of 42 nurses from 42 general hospitals. All participants worked in hospitals with computerized incident reporting systems, though the degree of computerization varied across hospitals.

\section{Data Collection Procedure}

Participants were asked to describe current issues in the operation of patient safety incident reporting systems in their hospitals and ways to resolve these issues using two openended questions on the barriers to and enablers of the operation of hospital incident reporting systems. We did not use additional questions that could reflect researcher's prejudice and lead to participants' induced responses for the purpose 
of this study. Data were collected via interview or email from July 2010 to April 2011. The explanation on the study purpose and research questions was given to the participants. Email was used for participants who want to reply the research questions via email instead of interview because of their busy schedule. However, to collect reliable data, if necessary, we had repeated contact with participants via email or face-to-face interview. Data on the general characteristics of participants-including age, education level, years of experience in hospital, and job position-were also collected. To facilitate frank commentary, we assured them of the confidentiality of their responses. Although we could not collect data anonymously, confidentiality was addressed by removing identifiers in the analysis set, including peer debriefing and peer scrutiny process.

\section{Data Analysis}

Data were analyzed using qualitative content analysis. To derive the major themes on the barriers to and measures for improvement of incident reporting, we used a framework of organizational and individual factors based on previous research [8]. The research framework consisted of 'organizational factors' and 'individual factors' in 'internal' and 'external' environment of hospitals regarding the barriers and measures to overcome such barriers, which was used as initial coding scheme (Figure 1). In this study, we classified educational and cultural aspects as organizational factors. Specifically, qualitative content analysis was conducted with following steps: 1) preparing the data: transcribing interviews and extracting responses to the open-ended questions; 2) defining the unit of analysis: representing a single theme or issue of relevance to our research questions; 3) coding the text: using a coding scheme based on the framework derived from the findings of previous related studies. Furthermore, to generate more granular categories, we used a constant comparison procedure. Text data were coded using

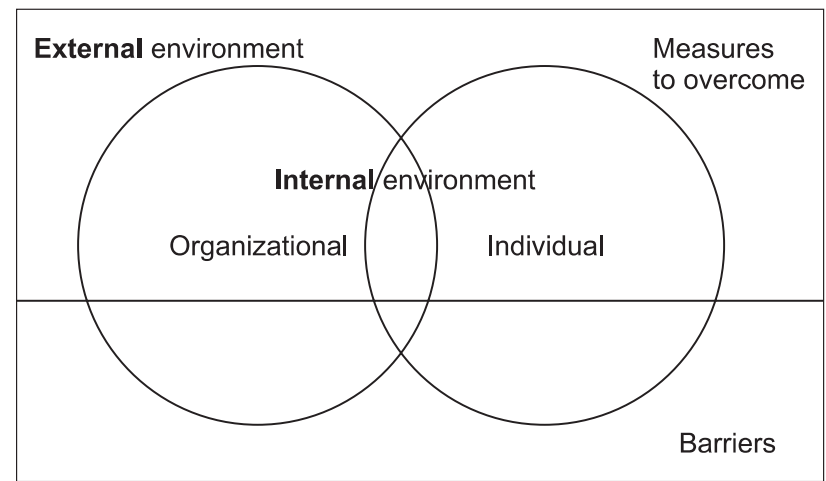

Figure 1. Research framework used in this study.
Microsoft Excel; 4) assessing the consistency and credibility of the coding: using repeated review through research team discussion; and 5) drawing conclusions from the coded data. During the process, the first author was responsible for the primary analysis and coding of the data. For peer scrutiny, the analysis results were validated by two co-authors as content and methodology experts. The general characteristics of respondents and the coded data were then summarized using descriptive statistics.

Table 1. General characteristics of participants

\begin{tabular}{lc}
\hline \multicolumn{1}{c}{ Variable } & No. (\%) \\
\hline Age $(\mathrm{yr})$ & $9(21.4)$ \\
$\leq 39$ & $29(69.0)$ \\
$40-49$ & $4(9.5)$ \\
$\geq 50$ & \\
Educational level & $9(21.4)$ \\
Doctoral program & $30(71.4)$ \\
Master program & $3(7.1)$ \\
Baccalaureate & \\
Experience in the hospital (yr) & $2(4.8)$ \\
$\leq 9$ & $18(42.9)$ \\
$10-19$ & $21(50.0)$ \\
$20-29$ & $1(2.4)$ \\
$\geq 30$ &
\end{tabular}

Job position

Manager

$40(95.2)$

Staff

Experience in current job (yr)

$<3$

3-5

6-10

18 (42.9)

$\geq 11$

Type of hospital ownership

Public

Private 34 (81.0)

Location

Metropolitan cities $29(69.0)$

Smaller cities

Bed size

$<500$

500-799

18 (42.9)

$\geq 800$

18 (42.9)

Total $42(100.0)$ 


\section{Results}

\section{General Characteristics of Participants}

The general characteristics of participants are given in Table 1. All of the participants $(n=42)$ were women, and $69.0 \%(n$ = 29) were between 40 and 49 years old. Over 90\% $(n=39)$ of the participants had master or doctoral degrees. All were nurses, and 95.2\% $(\mathrm{n}=40)$ held a managerial position. Most participants $(95.2 \%, \mathrm{n}=40)$ had been working ten years or more at the present hospital. Regarding their experience in working in incident reporting, $42.9 \%(n=18)$ had 5 to 9 years of experience, and $47.6 \%(n=20)$ had less than 5 years of experience. Of the hospitals where the participants worked, $81.0 \%(n=34)$ were private hospitals, and $69.0 \%$ were located in metropolitan cities. The mean number of beds was 828 (standard deviation $=377)$.

\section{Barriers to the Operation of Incident Reporting Systems}

Participants suggested 96 organizational and individual factors as barriers to incident reporting in their hospitals (Table 2). The most frequent problem they faced was lack of incident reporting, especially for near misses: "The first barrier is the omission of reporting incidents." (subjects 1-7), "The number of reporting near misses is particularly low." (subjects 8, 9).

The next two high-frequency categories included "constraints in the incident reporting system" and "issues with middle-level managers' leadership." In particular, the category of "constraints in the incident reporting system" included "no assurance of the anonymity of reporters" $(n=3)$, "dual reporting systems (e.g., reporters could report the in-

Table 2. Barriers in the operation of incident reporting systems

\begin{tabular}{|c|c|}
\hline Barriers & No. $(\%)$ \\
\hline Mainly organizational factors & $43(44.8)$ \\
\hline $\begin{array}{l}\text { Constraints of incident reporting systems (e.g., no assurance of anonymity, no integrated, dual reporting } \\
\text { systems; lack of system accessibility, usability problem; difficult to report multi-department involved } \\
\text { incidents) }\end{array}$ & $10(10.4)$ \\
\hline $\begin{array}{l}\text { Weak safety culture (e.g., blame and punishment for person involved in the incident; blame for depart- } \\
\text { ment involved in the incident) }\end{array}$ & $6(6.3)$ \\
\hline $\begin{array}{l}\text { Inter-department conflict and lack of cooperation (e.g., lack of cooperation from clinical departments } \\
\text { unavailable department-specific incident cases; conflict due to which department are responsible for the } \\
\text { incident) }\end{array}$ & $6(6.3)$ \\
\hline $\begin{array}{l}\text { Limited reporting (e.g., lack of reporting by the other department except nursing department; reporting } \\
\text { only incidents due to external factors such as caregivers) }\end{array}$ & $6(6.3)$ \\
\hline Intractable cases within time and financial constraints & $6(6.3)$ \\
\hline Absence of fulltime patient safety officers & $5(5.2)$ \\
\hline Delayed feedback & $2(2.1)$ \\
\hline Absence of education and training opportunities on patient safety and incident reporting & $2(2.1)$ \\
\hline Mainly individual factors & $53(55.2)$ \\
\hline Low reporting rate & $13(13.5)$ \\
\hline $\begin{array}{l}\text { Middle-level managers: lack of patient safety leadership (e.g., lack of awareness of the importance of patient } \\
\text { safety incident reporting lack of knowledge and skills of patient safety and incident reporting, fear of blame) }\end{array}$ & $10(10.4)$ \\
\hline Lack of physician's reporting and participation & $7(7.3)$ \\
\hline Top-level managers: lack of patient safety leadership & $6(6.3)$ \\
\hline $\begin{array}{l}\text { Staff: lack of knowledge and skill related to incident reporting (e.g., use of tools such as root cause analysis } \\
\text { and failure mode and effects analysis what should be reported and how to report) }\end{array}$ & $6(6.3)$ \\
\hline Lack of staff awareness of the importance of patient safety incident reporting & $5(5.2)$ \\
\hline Late reporting & $3(3.1)$ \\
\hline Fear of blame, stress & $2(2.1)$ \\
\hline Insufficient knowledge and skills related to incident reporting of risk managers & $1(1.0)$ \\
\hline Total & $96(100.0)$ \\
\hline
\end{tabular}


cident to either the nursing department or the patient safety management department, and the reports were less likely to be shared between the two departments)" ( $=3$ ), "lack of system accessibility (e.g., managing incidents occurring during holidays, requiring formal acceptance from middle level managers for reporting)" ( $\mathrm{n}=2$ ), "poor reporting process for problems involving more than one department" $(\mathrm{n}=1)$, and "problems in the usability of reporting systems" $(\mathrm{n}=1)$.

\section{Measures to Resolve or Overcome Problems or Barriers} From the responses of 41 participants, 104 measures to resolve or overcome their problems or barriers were derived (Table 3). The most frequently recommended measure was "utilizing rewards," followed by "improving reporting systems," "recruiting more staff for patient safety incident management," "enhancing the safety culture," and "providing education and training opportunities." In particular, measures to improve the reporting systems included allowing for various reporting channels (e.g., by computer, paper, email, or telephone, and utilizing different sources to identify the occurrence of incidents such as patient complaints and medical claims), assuring anonymity and confidentiality, revising incident report forms, employing a unified reporting system, and allowing integration with existing hospital information systems: "Providing incentives for reporting near misses will be useful"(subjects 1, 12), "It does not matter whether the reporting form is paper-based or computer-based. We should use various channels for reporting such as paper, telephone

Table 3. Measures to resolve or overcome barriers

\begin{tabular}{|c|c|}
\hline Measure & No. $(\%)$ \\
\hline External & $3(2.9)$ \\
\hline \multicolumn{2}{|l|}{ Need for education and training programs provided by academic society } \\
\hline \multicolumn{2}{|l|}{ Enforcement of patient safety standards in the Healthcare Accreditation Program } \\
\hline \multicolumn{2}{|l|}{ Establishment of a national institute to support hospitals' patient safety activity } \\
\hline Internal & $101(97.1)$ \\
\hline Organizational & $81(77.9)$ \\
\hline $\begin{array}{l}\text { Introducing a rewarding system (e.g., rewarding for near misses, department-level rewarding link to } \\
\text { individual performance appraisal) }\end{array}$ & $16(15.4)$ \\
\hline $\begin{array}{l}\text { Enhancing incident reporting systems (e.g., avariety of reporting channel: computer, paper, email, telephone } \\
\text { use of data from different sources such as patient complaints and malpractice claims improving system } \\
\text { accessibility using various platform in computers assurance of anonymity and confidentiality, improving } \\
\text { reporting forms integrated, unified reporting systems with existing hospital information systems) }\end{array}$ & $12(11.5)$ \\
\hline Enhancing safety culture & $11(10.6)$ \\
\hline Providing education and training opportunities & $11(10.6)$ \\
\hline Improving staffing for patient safety incident reporting management & $9(8.7)$ \\
\hline Visualizing and sharing successful results/outcomes & $7(6.7)$ \\
\hline Promotional activities (e.g., patient safety day ceremony, poster display) & $5(4.8)$ \\
\hline Providing feedback & $3(2.9)$ \\
\hline Designation of patient safety facilitators at the department level & $2(1.9)$ \\
\hline Monitoring and surveying safety culture & $1(1.0)$ \\
\hline Monitoring and surveying staff perception of patient safety & $1(1.0)$ \\
\hline Non-punitive policy & $1(1.0)$ \\
\hline Hospital-wide efforts to improve patient safety & $1(1.0)$ \\
\hline Creating a formal committee dealing with incident reports & $1(1.0)$ \\
\hline Individual & $20(19.2)$ \\
\hline Improving staff's awareness of patient safety and incident reporting & $9(8.7)$ \\
\hline Strengthening patient safety leadership from top-level managers & $7(6.7)$ \\
\hline Strengthening patient safety leadership from middle-level managers & $4(3.8)$ \\
\hline Total & $104(100.0)$ \\
\hline
\end{tabular}


especially for clinical department, email, as well as computerbased incident reporting systems" (subject 13).

\section{Discussion}

Incident reporting systems in hospitals provide valuable data and information for improving patient safety through analysis of the nature and patterns of incidents that have occurred. This study explored the current problems in the operation of incident reporting systems and high priority measures to overcome and resolve these problems.

The most frequently described problem with incident reporting systems was a low reporting rate, especially for near misses. This finding is consistent with those of previous studies in Korean hospitals, which found that nurses and physicians were uncomfortable with reporting errors $[12,13]$. Furthermore, it was similar with previous findings that near misses were the least reported types of incidents $[5,6,10]$. Near misses provide useful information on defects and recovery mechanisms in the care delivery system to prevent the recurrence of errors. The present study indicated that increasing overall reporting rates, including those of near misses, is a primary challenge that current incident reporting systems must overcome. These low reporting rates can be attributable to all the other organizational and individual factors that participants pointed out. These factors should be taken into account in the development of incident reporting systems.

In terms of organizational factors, the most frequently described barrier to incident reporting was associated with how the systems were designed. Because these incident reporting systems required reporters to identify themselves, participants were often dissuaded from reporting incidents. This problem was more prevalent in hospitals that had introduced computerized reporting systems. In addition, reporting systems had difficulty in integrating the reports that involved multiple departments and providing timely information to the person responsible for managing incident reporting. Leadership of top-level managers in the patient safety was also emphasized. Moreover, insufficient or partial integration between newly computerized reporting systems and conventional reporting systems seems to prevent efficient incident reporting in busy clinical situations. These problems indicate the necessity for standardized guidelines on system requirements in developing computerized incident reporting systems.

As for other barriers at the organizational level, an organizational cultural practice of blaming and penalizing the department heads as well as those involved in medical errors was frequently reported. This finding is similar to that in previous research showing that such an organizational culture negatively influenced staff members' incident reporting $[4,15]$. Other perceived barriers included interdepartment conflict and lack of interdepartment cooperation, incident reporting being used only in the nursing department, and intractable cases that require complex measures for improvement. In particular, the lack of physicians' participation in incident reporting was described as a problem. This finding is similar to other studies that suggested incident reporting was used predominantly by nurses [5]. These organizational issues should be taken into account when designing computerized reporting systems to improve their usability and acceptability.

Regarding the individual barriers to incident reporting, participants perceived that a major barrier was associated with the role of middle-level managers. Department managers can control reporting processes by accepting or rejecting a specific incident report in a formal reporting line. Their awareness and attitude towards incident reporting directly influences the reporting practices of their staff. Therefore, incident reporting systems need to be independent of any authority with power to punish the reporter or organization [16,17]. In addition, most hospital staff lack the knowledge and skills required for effective incident reporting (e.g., knowledge of sentinel events and near misses). This finding is similar to a previous report, which indicated that a lack of understanding about what constitutes a patient safety incident is a major barrier to incident reporting [10]. Furthermore, participants reported that staff lacked the skill for analyzing incidents to determine their root causes. Therefore, templates that would aid in the collection of data when incidents are being reporting should be designed and implemented in hospital reporting systems.

Participants suggested several measures to overcome these barriers and implement successful incident reporting systems. To facilitate staff reporting, including near misses, many participants proposed introducing a rewards system. However, participants who were working in hospitals where a rewards system was already in place, either at the individual or department levels, pointed out that rewards should be given only on a case-by-case basis, because in cases where the incident is clearly the fault of an individual, positive rewards would be inappropriate. Regarding improvements to the reporting systems themselves, the assurance of anonymity and confidentiality when reporting incidents was considered important. Moreover, the use of a variety of reporting channels was recommended for more active incident reporting, such as paper-based reporting, stand-alone or integrated 
computer-based reporting, and email or telephone reporting, as was the use of different sources to identify the occurrence of incidents, including patient complaints or malpractice claims. In addition, participants emphasized the enhancement of the safety culture in their hospital, which would necessitate continuous, organization-wide efforts. One participant suggested the need to diagnose and monitor the patient safety culture. Strong safety culture provides a basis for safer performance by encouraging adherence to effective safety practices as well as learning from errors. An important characteristic of a safety culture is blame-free environment for reporting [18]. A systematic review concluded that leadership walk rounds and multi-faceted unit-based programs may have a positive impact on patient safety climate [19]. Therefore, these actions are recommended to improve patient safety culture. In addition to improving managers' patient safety leadership, strategies to overcome low reporting rates included providing education and training opportunities to staff to improve their knowledge and skills regarding patient safety and incident reporting systems, and sharing and discussing the best practices for improving patient safety. Therefore, the scope of incident reporting systems needs to be expanded to implement such needs.

However, the present study has certain limitations. First, the study was conducted in only 42 general hospitals. Thus, the generalizability of the findings is limited. However, we included $80 \%$ of all Korean tertiary hospitals (35 out of 44 ). Since they have played a leading role in the Korean healthcare system, their experience in the operation of computerized incident reporting systems can provide practical implications. Second, this study did not include responses from frontline healthcare providers, who might have different perspectives from our participants. Therefore, future studies are needed with frontline users of incident reporting systems as study participants in various hospital settings. In addition, we did not perform member checking to allow participants to determine if the findings reflect their experiences because of practical difficulties. Instead, we used peer scrutiny along with methodological triangulation to assure credibility of the findings.

In conclusion, to support valid and reliable findings, we used methodological triangulation. In addition, the consistency and credibility of the coding was reviewed through a peer scrutiny. Overall, this study identified a number of organizational and individual factors that act as barriers to incident reporting practices in general hospitals. Low reporting rates, especially for near misses, was a primary issue. Furthermore, the poor design of incident reporting systems, including a lack of reporter anonymity, was the most fre- quently perceived barrier to incident reporting. The other major challenge to the operation of incident reporting systems is related to the poor leadership of middle-level managers. To resolve and overcome these barriers, and improve the systems overall, a number of efforts should be made at the individual and organizational levels. High-priority measures included introducing reward systems, improving incident reporting systems by including a variety of reporting channels and ensuring reporter anonymity, and creating a strong safety culture within the hospital.

These findings provide basic data on the high-priority areas that must be addressed for successful implementation of better incident reporting systems in hospitals. In particular, this study emphasized the importance of design of hospital incident reporting systems and role of middle level managers. These challenges also need to be addressed when hospitals tried to integrate their incident reporting systems with existing hospital information system for more efficient data gathering and analysis, along with leadership development for middle level managers on patient safety. Furthermore, we would like to suggest that future research should investigate how various interfaces employed in incident reporting systems influence staff members' reporting behavior.

\section{Conflict of Interest}

No potential conflict of interest relevant to this article was reported.

\section{Acknowledgments}

This research was supported by Basic Science Research Program through the National Research Foundation of Korea (NRF) funded by the Ministry of Education, Science and Technology (project no. 2010-0006454).

\section{References}

1. Kohn LT, Corrigan J, Donaldson MS. To err is human: building a safer health system. Washington (DC): National Academy Press; 2000.

2. Henriksen K, Battles JB, Marks ES, Lewis DI. Advances in patient safety: from research to implementation. Rockville (MD): Agency for Healthcare Research and Quality; 2005.

3. Kousgaard MB, Joensen AS, Thorsen T. Reasons for not reporting patient safety incidents in general practice: a qualitative study. Scand J Prim Health Care 2012;30(4): 199-205. 
4. Braithwaite J, Westbrook MT, Travaglia JF, Hughes C. Cultural and associated enablers of, and barriers to, adverse incident reporting. Qual Saf Health Care 2010; 19(3):229-33.

5. Evans SM, Berry JG, Smith BJ, Esterman A, Selim P, O'Shaughnessy J, et al. Attitudes and barriers to incident reporting: a collaborative hospital study. Qual Saf Health Care 2006;15(1):39-43.

6. Firth-Cozens J. Barriers to incident reporting. Qual Saf Health Care 2002;11(1):7.

7. Lawton R, Parker D. Barriers to incident reporting in a healthcare system. Qual Saf Health Care 2002;11(1):15-8.

8. Uribe CL, Schweikhart SB, Pathak DS, Dow M, Marsh GB. Perceived barriers to medical-error reporting: an exploratory investigation. J Healthc Manag 2002;47(4): 263-79.

9. Edmondson AC. Learning from failure in health care: frequent opportunities, pervasive barriers. Qual Saf Health Care 2004;13 Suppl 2:ii3-9.

10. Kreckler S, Catchpole K, McCulloch P, Handa A. Factors influencing incident reporting in surgical care. Qual Saf Health Care 2009;18(2):116-20.

11. Haller G, Courvoisier DS, Anderson H, Myles PS. Clinical factors associated with the non-utilization of an anaesthesia incident reporting system. Br J Anaesth 2011; 107(2):171-9.

12. Kang M, Kim JE, An KE, Kim Y, Kim SW. Physicians' perception and attitude towards patient safety culture and medical error reporting. Korean J Health Policy Adm 2005;15(4):110-35.

13. Kim JE, Kang M, An KE, Sung YH. A survey of nurses' perception of patient safety related to hospital culture and reports of medical errors. J Korean Clin Nurs Res 2007;13(3):169-79.

14. Kim J, Kim S, Jung Y, Kim EK. Status and problems of adverse event reporting systems in Korean hospitals. Healthc Inform Res 2010;16(3):166-76.

15. El-Jardali F, Dimassi H, Jamal D, Jaafar M, Hemadeh N. Predictors and outcomes of patient safety culture in hospitals. BMC Health Serv Res 2011;11:45.

16. Leape LL. Reporting of adverse events. N Engl J Med 2002;347(20):1633-8.

17. Harper ML, Helmreich RL. Identifying barriers to the success of a reporting system. In: Henriksen K, Battles JB, Marks ES, Lewis DI. Advances in patient safety: from research to implementation. Rockville (MD): Agency for Healthcare Research and Quality; 2005.

18. Alahmadi HA. Assessment of patient safety culture in Saudi Arabian hospitals. Qual Saf Health Care 2010;19(5):e17.

19. Morello RT, Lowthian JA, Barker AL, McGinnes R, Dunt D, Brand C. Strategies for improving patient safety culture in hospitals: a systematic review. BMJ Qual Saf 2013;22(1):11-8. 